\title{
Parton Distributions for LO Calculations
}

\author{
R.S. Thorne ${ }^{1 *}$ A. Sherstnev ${ }^{2}$ and C. Gwenlan ${ }^{1}$ \\ 1- Department of Physics and Astronomy, University College London, WC1E 6BT, UK \\ 2- Cavendish Laboratory, University of Cambridge, Cambridge, CB3 0HE, UK
}

\begin{abstract}
We present a study of the results obtained combining LO partonic matrix elements with different orders of partons distributions. These are compared to the best prediction using NLO for both matrix elements and parton distributions. The aim is to determine which parton distributions are most appropriate to use in those cases where only LO matrix elements are available, e.g. as in many Monte Carlo generators. Both LO and NLO parton distributions have faults so a modified optimal LO set is suggested.
\end{abstract}

The combination of the order of the parton distribution function (pdf) and the accompanying matrix element is an important issue [1]. It has long been known that LO pdfs in some regions of $x$ and $Q^{2}$ are qualitatively different to NLO (and NNLO) pdfs (see [2]) due to important missing corrections in splitting functions or coefficient functions for structure functions which are used to determine the pdfs. Nevertheless, LO pdfs are usually thought to be the best choice for use with LO matrix elements, such as those available in many LO Monte Carlo programs, though all such results should be treated with care. Recently it has been suggested that NLO pdfs may be more appropriate [3], since NLO cross-section corrections are often small. There has already been an investigation of the use of NLO pdfs for the underlying event [4]. There is a big difference in the results when using CTEQ6L and CTEQ6.1M pdfs [5] due to the changes in the gluon, though agreement can be reached by significant retuning. This will affect predictions for other quantities.

In this article we address the differences in predictions obtained for a variety of physical quantities combining different pdfs with LO matrix elements. In each case NLO pdfs combined with NLO matrix elements represent the best prediction - the truth. We interpret the features of the results and investigate
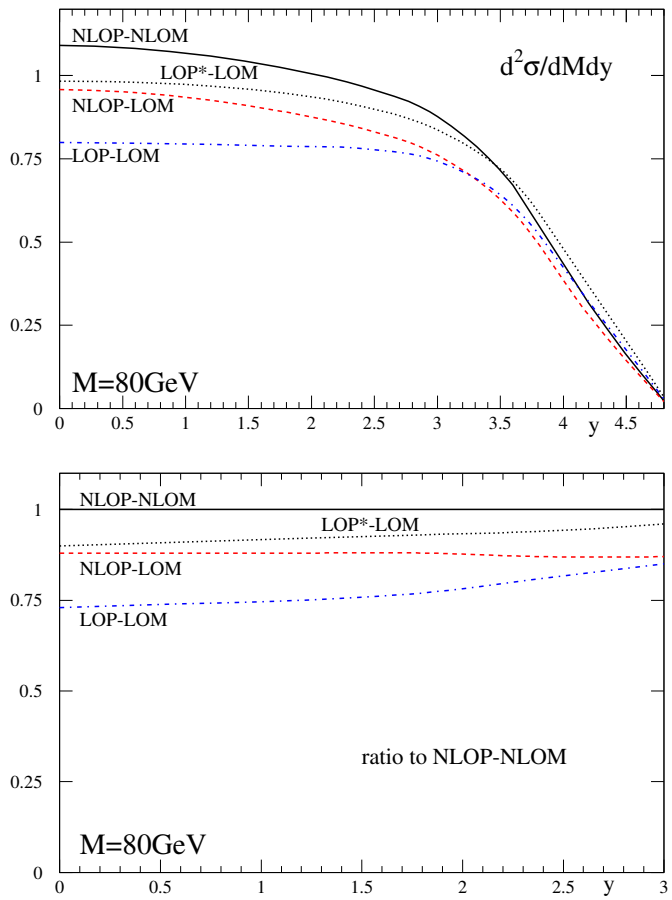
how a best set of pdfs for use with LO matrix elements may be obtained.

First, let us recall how LO pdfs tend to differ from NLO pdfs. The most marked differences are for light quarks at high $x$ and the gluon distribution at low $x$. The coefficient functions for structure functions have $\ln (1-x)$ enhancements at higher perturbative order. This means the high- $x$ quarks are smaller as the order increases. The quark-gluon splitting function $P_{q g}$ develops a small- $x$ divergence at NLO (with further $\ln (1 / x)$ enhancements at

${ }^{*}$ Royal Society University Research Fellow 
higher orders), so the small $x$ gluon needs to be bigger at LO in order to fit structure function evolution. Indeed, for $Q^{2} \sim 1-2 \mathrm{GeV}^{2}$ the NLO gluon is flat or valence-like at small $x$ while at LO it grows much more quickly. These features show up in cross-sections.

Let us start with the simple example of Drell-Yan production of vector-bosons from quark-antiquark annihilation as a function of rapidity at fixed invariant mass $=80 \mathrm{GeV}$, i.e. appropriate for $W, Z$ production, at the LHC. The NLO correction [6] for Drell-Yan production is quite significant, being positive for all rapidity and roughly $12 \%$ in this case. The absolute predictions and the ratios of LO matrix element results to the truth are show in Fig. 1. We see that indeed we are nearer to the truth with LO matrix elements and NLO pdfs [7] than LO matrix elements and LO pdfs [8]. Both LO ME results are too small, but NLO pdfs are closer and a better shape. LO pdfs and the LO matrix elements have the wrong shape being low at central rapidity but increasing at high rapidity where the high- $x$ quark is enhanced at LO. Hence, NLO pdfs seem more appropriate here.

However, there is a small $x$ counterexample. Consider the production of charm in low $Q^{2}$ DIS at HERA energies. The NLO matrix elements [9] contain a divergence at small $x$ which is not present at LO. Using NLO pdfs the LO matrix element result is well below the truth at low scales and the shape is totally wrong, as seen in Fig. 2. The LO gluon is very large at small $x$ since it has been extracted with missing $P_{q g}$ enhancement at small $x$, and this compensates the missing small $x$ divergence in the matrix element - LO pdfs and LO matrix elements are more sensible with a compensation between failings in both. Using LO pdfs and NLO matrix elements the result is extremely large since there is a double counting of the small $x$ divergence.

From these two examples we can conclude that sometimes NLO pdfs are better to use if only the LO matrix elements are known, and we can get significant problems with the size and shape if LO pdfs are used. However, we can be completely wrong, particularly at
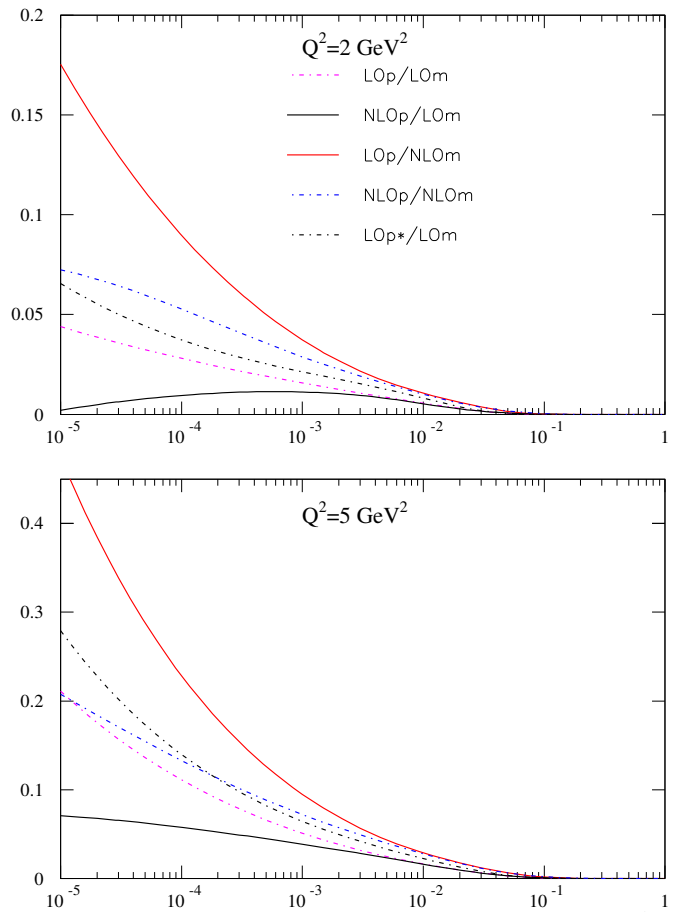

Figure 2: The structure function $F_{2}^{c}\left(x, Q^{2}\right)$. small $x$, if we use NLO pdfs due to zero-counting of small- $x$ terms. Can we find some optimal definition of pdfs which have most desirable features? In order to make progress we need to better understand the difference between LO and NLO pdfs. The missing higher order terms in $\ln (1-x), 1 / x$ and $\ln (1 / x)$ in coefficient functions and/or evolution leads to pdfs at LO which are bigger at $x \rightarrow 1$ and at $x \rightarrow 0$ in order to compensate. However, from the momentum sum rule there are then not enough partons to go around, and enhancements in some regions lead to depletion in other regions, particularly quarks at $0.001 \leq x \leq 0.1$. This leads to a bad global fit at LO [8] - partially compensated by the LO extraction of $\alpha_{S}\left(M_{Z}^{2}\right)$ being $\sim 0.130$ to help speed evolution, and explains the underestimate of the Drell-Yan production at the LHC at more central rapidities.

This obvious source of problems has lead to a suggestion [10] that relaxing the momentum 
sum rule could make LO pdfs rather more like NLO pdfs in the regions where they are normally too small. The resulting pdfs would still be bigger than NLO where necessary, i.e. the high- $x$ quarks and low- $x$ gluon, but would not be depleted elsewhere. It is also useful to use the NLO definition of $\alpha_{S}$. Because of quicker running at NLO, the LO and NLO couplings with the same value of $\alpha_{S}\left(M_{Z}^{2}\right)$ are very different at lower scales where DIS data used in global fits exists. Near $Q^{2}=1 \mathrm{GeV}^{2}$ the NLO coupling with $\alpha_{S}\left(M_{Z}^{2}\right)=0.120$ is similar to the LO coupling with $\alpha_{S}\left(M_{Z}^{2}\right)=0.130$. Hence, the use of the NLO coupling helps alleviate the discrepancy between pdfs at different orders. Indeed, the NLO coupling already used in some CTEQ LO pdfs [5] and in LO Monte Carlo generators. Relaxing momentum conservation for input pdfs and using the NLO definition of $\alpha_{S}$ does dramatically improve the quality of the LO global fit, $\chi^{2}=3066 / 2235$ for the standard LO fit becoming $\chi^{2}=2691 / 2235$, with a big improvement in the comparison to HERA data. The momentum carried by the input pdfs goes up to $113 \%$. Using the NLO definition the value of $\alpha_{S}\left(M_{Z}^{2}\right)=0.121$.

The modified pdfs, which we denote by $\mathrm{LO}^{*}$, become much more similar to NLO pdfs, in particular at small $x$ the LO* quark distributions evolve as quickly as at NLO and are similar for for $x \sim 0.001-0.01$. Similarly $g\left(x, Q^{2}\right)$ is significantly bigger at $\mathrm{LO}^{*}$ than at $\mathrm{LO}$, and much bigger than NLO at small $x$. This will help when used with LO matrix elements for gluon-gluon initiated processes (e.g. Higgs production) where $K$-factors are often much greater

\begin{tabular}{|c|c|c|}
\hline parton & $\begin{array}{c}\text { matrix } \\
\text { element }\end{array}$ & $\sigma(\mathrm{mb})$ \\
\hline NLO & NLO & 41.5 \\
LO & LO & 24.8 \\
LO* & LO & 34.8 \\
NLO & LO & 16.8 \\
\hline
\end{tabular}

Table 1: $\sigma(b \bar{b})$ totals. than unity. We now look at the LO* pdfs in our first two examples, see Figs. 1 and 2. For Drell-Yan production at the LHC the LO* pdfs lead to shape of comparable quality as the NLO pdfs and the normalization is better. For the charm structure function comparing all possibilities the LO* pdfs and LO matrix elements are indeed closest to the truth.

There is a similar conclusion for hadro-production of $b$ quarks at LHC which probes low $x \sim 0.001$. We consider $\sigma(b \bar{b})$ where the initial $b$ quark has $p_{T}>10 \mathrm{GeV}$ and $|\eta| \leq 5$ using a NLO event generator [11] and LO calculations [12]. The total cross-sections are shown in Table. 1. The NLO pdfs and LO matrix element are clearly worst. We also illustrate final state $p_{T}$ and rapidity distributions in Fig. 3 with $p_{T}>18$ $\mathrm{GeV}$ for the $b$ quark after showering. Again the best absolute predictions with LO matrix elements uses $\mathrm{LO}^{*}$ pdfs and the worst NLO
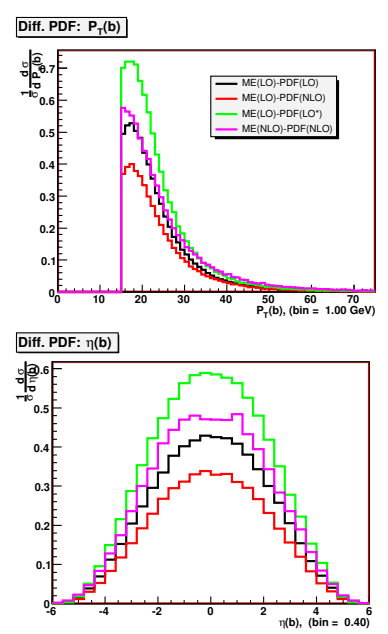

Figure 3: The $b$ cross section at the LHC. pdfs. However, in this case there is always a problem with the shape as function of $p_{T}$. The NLO matrix element has a large positive effect at high $p_{T}$ and very high $\eta$. It is impossible for any parton shape to account for all NLO corrections.

We also look at very high- $E_{T}$ jet production [13] at the LHC in Fig. 4. Ignoring the lowest $E_{T}$, where hadronization and underlying event and possibly small $x$ physics are an issue, 
the $\mathrm{LO}$ and $\mathrm{LO}^{*}$ pdfs with the LO matrix elements are of comparable quality to NLO pdfs. LO and NLO pdfs when combined with LO matrix elements produce results which deviate in opposite directions at high $E_{T}$. Also shown is the prediction from $\mathrm{LO}^{* *}$ pdfs, which have an additional constraint for a harder high- $x$ gluon (this being small at LO even with momentum conservation relaxed). This changes the results little compared to the use of the $\mathrm{LO}^{*}$ pdfs.

Hence, we can conclude that a fixed prescription of either LO or NLO pdfs with LO matrix elements or LO Monte Carlo generators will lead each to incorrect results in some cases. To try to improve this situation we have suggested an optimal set of pdfs for LO calculations, the LO* pdfs, which are essentially LO but with various modifications to make their features more NLOlike. These seem to work reasonably well and happen to achieve some of the features obtained by modifying pdfs in a process dependent fashion for use in Monte Carlo gen-

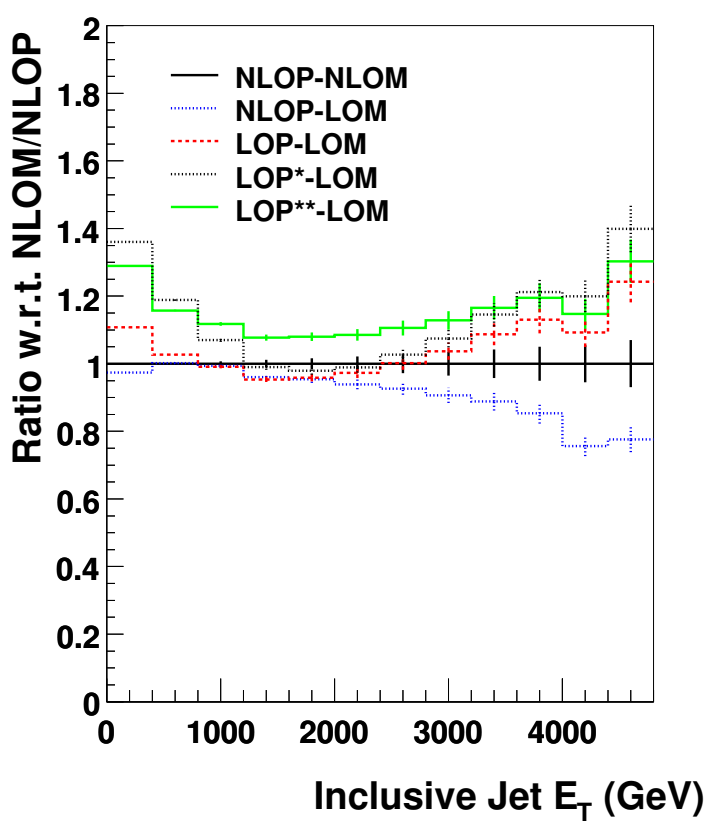

Figure 4: The dijet cross-section at the LHC. erators/resummations discussed in e.g. [14]. More study is underway. However, sometimes NLO matrix element corrections qualitatively change the features of the predictions for a physical process, regardless of how careful one is with pdfs, since new types of partonic process open up. This must always be borne in mind, and accounted for if possible.

\section{References}

[1] Slides: http://indico.cern. ch/contributionDisplay. py? contribId=252\&sessionId=8\&conf Id=9499

[2] R. S. Thorne, plenary talk at DIS06, Tsukuba, Japan, 20-24 Apr 2006, pp.35, hep-ph/0606307.

[3] J. M. Campbell, J. W. Huston and W. J. Stirling, Rept. Prog. Phys. 70 (2007) 89.

[4] Section by R. Field in M. G. Albrow et al. [TeV4LHC QCD Working Group], "Tevatron-for-LHC report of the QCD working group," hep-ph/0610012.

[5] J. Pumplin, D. R. Stump, J. Huston, H. L. Lai, P. Nadolsky and W. K. Tung, JHEP 0207 (2002) 012.

[6] J. Kubar, M. Le Bellac, J. L. Meunier and G. Plaut, Nucl. Phys. B 175 (1980) 251.

[7] A.D. Martin, R.G. Roberts, W.J. Stirling and R.S. Thorne, Phys. Lett. B604 61 (2004).

[8] A. D. Martin, R.G. Roberts, W.J. Stirling and R.S. Thorne, Phys. Lett. B 531 (2002) 216.

[9] E. Laenen et al., Nucl. Phys. B392 (1993) 162; B.W. Harris and J. Smith, Nucl. Phys. B452 (1995) 109.

[10] T. Sjöstrand, private comments.

[11] S. Frixione and B.R. Webber, JHEP 0206 (2002) 029; S. Frixione et al., JHEP 0308 (2003) 007.

[12] E.Boos et al. [CompHEP Collaboration], Nucl. Instrum. Meth. A534 (2004) 250; A.S. Belyaev et al., hep-ph/0101232; T. Sjöstrand, S. Mrenna and P. Skands, JHEP 0605 (2006) 026.

[13] W.T. Giele, E.W.N. Glover and D.A. Kosower, Nucl.Phys. B403 (1993) 633.

[14] S. Mrenna, hep-ph/9902471. 